\title{
INVERSION OF A NON-UNIFORM DIFFERENCE OPERATOR
}

\author{
BLAKE TEMPLE AND ROBIN YOUNG
}

\begin{abstract}
The problem of applying Nash-Moser Newton methods to obtain periodic solutions of the compressible Euler equations has led authors to identify the main obstacle, namely, how to invert operators which impose periodicity when they are based on non-uniform shift operators. Here we begin a theory for finding the inverses of such operators by proving that a scalar non-uniform difference operator does in fact have a bounded inverse on its range. We argue that this is the simplest example which demonstrates the need to use direct rather than Fourier methods to analyze inverses of linear operators involving nonuniform shifts.
\end{abstract}

\section{INTRODUCTION}

In this article we explicitly invert the operator which imposes periodicity for a scalar shift, namely,

$$
\Delta_{\Phi} v:=\mathcal{S}_{\Phi} v-v=w
$$

where the shift operator $\mathcal{S}_{\Phi}$ is defined by

$$
\mathcal{S}_{\Phi} v=v \circ \Phi, \quad \text { so that } \mathcal{S}_{\Phi} v(t)=v(\Phi(t)),
$$

and where the non-uniform shift $\Phi(t)$ has the form

$$
\Phi(t)=t+\alpha \phi(t)
$$

We call $\phi$ the perturbation and $\alpha$ the amplitude, and say the perturbation is non-degenerate if it is Lipschitz continuous, and satisfies the further conditions

$$
\phi^{\prime}\left(t_{*}\right) \neq 0 \quad \text { whenever } \quad \phi\left(t_{*}\right)=0,
$$

and if at any zero $t_{*}$ of $\phi$, we have the Taylor estimate

$$
\phi(t)-\phi\left(t_{*}\right)-\phi^{\prime}\left(t_{*}\right)\left(t-t_{*}\right)=O\left(\left(t-t_{*}\right)^{2}\right) .
$$

This is the condition that $\phi$ be better than differentiable, but not quite twice differentiable, at each zero. In particular, any perturbation $\phi$ which is approximately sinusoidal is non-degenerate.

We introduce the problem of inverting $\mathcal{S}_{\Phi}-\mathcal{I}$ as a warmup problem for inverting the linearized operators associated with the nonlinear operators in [4, 5, 6, 7], which impose periodicity for the compressible Euler equations. In this case genuine nonlinearity enters as $\phi(t)=\sin (t)+O(\alpha)$ for small

Date: April 26, 2022. 
$\alpha$. For motivation, note that the time one evolution map of the nonlinear Burgers equation

$$
u_{t}+u u_{x}=0
$$

is a non-uniform shift of the form (1), so a time-periodic solution would satisfy $\Delta_{\Phi} v=0$. Since Burgers admits no smooth time-periodic solutions, we expect the linear difference operator $\Delta_{\Phi}=\mathcal{S}_{\Phi}-\mathcal{I}$ to admit no smooth periodic solutions as well. Here we address the question as to whether the inverse $\Delta_{\Phi}^{-1}$ is bounded. In [4, 5 , the authors derive a $2 \times 2$ linearized system of the form $\mathcal{S}_{\Phi}-\mathcal{J}$, where $\mathcal{S}_{\Phi}$ is a diagonal shift operator and $\mathcal{J}$ is a fixed constant linear operator; in this case, the shifts have the form

$$
\phi(t)=\sin (t)+O(\alpha), \quad \text { so that } \Phi(t)=t+\alpha \sin (t)+O\left(\alpha^{2}\right) .
$$

Inversion of the operator $\mathcal{S}_{\Phi}-\mathcal{J}$ is a critical step in the proof of existence of space- and time-periodic solutions of the compressible Euler equations by Nash-Moser methods, but the requisite estimates for the inverses of such operators involving shifts is beyond current mathematical technology. The authors propose the analysis of $\Delta_{\Phi}$ here as the first step in a program to develop a mathematical framework for inverting linearized shift operators in general. The equation $\Delta_{\Phi} v=0$ is an example of an iterative functional equation such as those treated in [1] from a different point of view.

When $\phi(t)=0, t$ is a rest point of $\Phi$, and it follows that the interval between any two consecutive roots of $\phi(t)$ is mapped to itself under $\Phi$, provided $\alpha$ is small enough. Denoting these roots by $t_{ \pm \infty}$, it follows that

$$
\phi\left(t_{-\infty}\right)=0=\phi\left(t_{+\infty}\right), \quad \text { and } \phi(t) \neq 0, \quad t \in\left(t_{-\infty}, t_{+\infty}\right),
$$

and in particular $\Phi:\left[t_{-\infty}, t_{+\infty}\right] \rightarrow\left[t_{-\infty}, t_{+\infty}\right]$, and

$$
\mathcal{S}_{\Phi}: L^{\infty}\left[t_{-\infty}, t_{+\infty}\right] \rightarrow L^{\infty}\left[t_{-\infty}, t_{+\infty}\right] .
$$

Thus to isolate the essential issue, we address the case when the domain of $t$ is the interval $\left[t_{-\infty}, t_{+\infty}\right]$, and $\Phi$ is a monotonic map of this interval to itself. We introduce a framework to invert the operator $\mathcal{S}_{\Phi}-\mathcal{I}$ on its range in subspaces of $L^{\infty}\left[t_{-\infty}, t_{+\infty}\right]$. In particular, we derive estimates for the inverse of $\Delta_{\Phi}$ in $C^{p}\left[t_{-\infty}, t_{+\infty}\right]$, the subspace of $L^{\infty}\left[t_{-\infty}, t_{+\infty}\right]$ consisting of functions with $p$ continuous derivatives, under the assumption that $\alpha$ is sufficiently small.

We begin by proving that the operator $\mathcal{S}_{\Phi}-\mathcal{I}$ admits a large class of solutions in $L^{\infty}\left[t_{-\infty}, t_{+\infty}\right]$ in its kernel, and we characterize them. However, if we require continuity at either endpoint $t=t_{ \pm \infty}$, we show that there are no non-constant elements of the kernel. We then obtain a formula for the inverse $\Delta_{\Phi}^{-1}$ by directly solving $\mathcal{S}_{\Phi} v-v=w$ for $v$ in terms of $w$. The resulting formula leads to a solvability condition for $w$, namely,

$$
\left|\sum_{k \geq 0} w\left(\Phi^{k} t\right)\right|<\infty, \quad\left|\sum_{k \geq 0} w\left(\Phi^{-k} t\right)\right|<\infty, \quad \sum_{k} w\left(\Phi^{k} t\right)=\text { Const },
$$


for every $t \in\left[t_{-\infty}, t_{+\infty}\right]$. We show that condition (15) characterizes the image of $\mathcal{S}_{\Phi}-\mathcal{I}$, and the image of $\Delta_{\Phi}$ in the space of Lipshitz continuous functions consists of precisely those Lipshitz continuous functions $w$ that meet condition (5). Moreover, we prove a bound on the inverse of $\mathcal{S}_{\Phi}-\mathcal{I}$. We denote the space of Lipshitz continuous functions by $C^{0,1}\left[t_{-\infty}, t_{+\infty}\right]$, with norm

$$
\|v\|_{C^{0,1}}=\|v\|_{\text {Lip }}+\|v\|_{\text {Sup }}
$$

where $\|v\|_{\text {Lip }}$ is the minimal Lipshitz constant. As long as the perturbation $\phi$ is Lipshitz, the map $\Delta_{\Phi}=\mathcal{S}_{\Phi}-\mathcal{I}$ is a bounded map from $C^{0,1}\left[t_{-\infty}, t_{+\infty}\right]$ to itself, and the following theorem describes its inverse.

Theorem. Assume that $\phi(t)$ satisfies (3) and (4). Then there exist constants $\alpha_{\phi}$ and $K_{\phi}$, given explicitly in terms of $\phi$, such that, if $\alpha<\alpha_{\phi}$ and $w \in C^{0,1}\left[t_{-\infty}, t_{+\infty}\right]$ satisfies $w\left(t_{-\infty}\right)=w\left(t_{+\infty}\right)=0$ together with the solvability condition (5), then the equation

$$
\Delta_{\Phi} v(t)=v(\Phi t)-v(t)=w(t)
$$

has a solution $v \in C^{0,1}\left[t_{-\infty}, t_{+\infty}\right]$, uniquely determined up to constant, which satisfies

$$
\|v\|_{\text {Lip }} \leq K_{\phi}\|w\|_{\text {Lip }}, \quad \text { so that } \quad\left\|\Delta_{\Phi}^{-1}\right\|_{\text {Lip }} \leq K_{\phi} .
$$

The theorem shows that $\Delta_{\Phi}: C^{0,1} \rightarrow C^{0,1}$ has a bounded inverse on its range, which consists of those Lipshitz functions satisfying the solvability condition. In Section 4 we prove that this result extends to invertibility in the space $C^{p}, p \in \mathbb{N}$, for $\alpha$ sufficiently small, c.f. Theorem 4 below. Indeed, we show that there exist constants $\alpha_{0}>0$ and $K$ depending only on $\phi$ and $p$, such that

$$
\left\|\Delta_{\Phi}^{-1} w\right\|_{p} \leq \frac{K}{|\alpha|}\|w\|_{p}, \quad \text { for } \quad|\alpha| \leq \alpha_{0}
$$

The solvability condition is essential because we obtain two apparently independent expressions for $v$ in terms of $w$, one of which converges, while the other need not. Our formula requires forward and/or backward iteration of the shift $\Phi$, and it is not a priori clear that both iterations converge. The solvability condition is then a compatibility between the two expressions, which yields a unique answer, and provides convergence in all cases.

Writing $\Delta_{\Phi}=\mathcal{S}_{\Phi}-\mathcal{I}$, it is insightful to compare $\Delta_{\Phi}$, a linear difference operator, to the derivative operator $D$. For the derivative operator, we know that the range, say on $C^{p}$, lies in the much larger space $C^{p-1}$, so $D$ can only be inverted on a subspace of $C^{p-1}$ which contains less regular functions (by one order) than its domain. In contrast, the difference operator $\Delta_{\Phi}$ has a range equal to a subset of the space of all Lipschitz functions, which is in fact the domain of $\Delta_{\Phi}$. So the difference operator is much more well behaved than the derivative operator. Nonetheless, the standard way of estimating the inverse of $\Delta_{\Phi}$ fails precisely because $\Delta_{\Phi} v$ does not bound $D v$. Assume for example that $\mathcal{S}_{\Phi}$ and $D$ are defined on $C^{p}$. Here is how a standard argument for estimating the inverse would go: 
Let $\mathcal{D}: C^{p} \rightarrow C^{p}$ be a general operator we would like to invert, and assume $v=0$ is the only solution to $\mathcal{D} v=0$; We'd like to prove

$$
\|v\|_{C^{p}} \leq \text { Const. }\|\mathcal{D} v\|_{C^{p}} .
$$

Assume not, so there exist $v_{k}$, which we can assume have unit length, such that

$$
1=\left\|v_{k}\right\|_{C^{p}}>k\left\|\mathcal{D} v_{k}\right\|_{C^{p}}, \quad \text { so that }\left\|\mathcal{D} v_{k}\right\|_{C^{p}} \rightarrow 0 .
$$

Now assume (and here is the point at which the argument fails for $\Delta_{\Phi}$ ) that $\mathcal{D}$ dominates the derivative in the sense that

$$
\|\mathcal{D} v\|_{C^{p}} \geq\|D v\|_{C^{p}} \equiv\|v\|_{C^{p+1}} .
$$

Then we would have $\left\|D v_{k}\right\|_{C^{p}}$ uniformly bounded, and this together with the bound $\left\|v_{k}\right\|_{C^{p}}=1$ would imply that $v_{k}$ is compact in $C^{p}$, and hence a subsequence $v_{k} \rightarrow \bar{v}$ would converge in $C^{p}$, and the limit would not be zero. But (8) implies that $\mathcal{D} \bar{v}=0$, contradicting the assumption that the $\mathcal{D} v=0$ has only the zero solution. Thus for this argument, we need to establish (8) in order to obtain a bound on the inverse of $\mathcal{D}$, but for shift operators, the estimate (8) clearly fails! The purpose of this note is to develop methods sufficient to prove the desired estimate (77) for the inverse of $\Delta_{\Phi}$ in this case when (8) fails.

The theorem as stated above gives an estimate for the bound on the inverse of $\Delta_{\Phi}$ in the Lipschitz norm for shift operators $\Phi$ that meet the admissibility conditions (3) and (44). The constant that bounds the inverse is $K_{\phi}$, defined in (30) below, and this depends on seven parameters, as expressed in Corollary 4 below. Although complicated, the main point is that the constant $K_{\phi}$ depends continuously on the norm of $\phi$ for any norm in which these seven parameters are continuous, such as say $C^{2}$. Similarly, the estimate (6) holds if $\phi \in C^{p+1}$, and the constants $\alpha_{0}$ and $K$ depend on $\|\phi\|_{C^{p+1}}$.

Part of the problem of obtaining bounds on the inverses of non-uniform difference operators is that the difference between two shift operators is an operator that loses a derivative [2, 8]. As a result, it is not possible to estimate a nearby linearized operator by an estimate for the unperturbed operator plus an estimate for the difference in the same norm. However, our results here show that estimates for the inverse of $\Delta_{\Phi}$ in $C^{p}$ are nonetheless stable under perturbation of $\phi$ in $C^{p+1}$ because they depend on constants which depend continuously on the $C^{p+1}$ norm of $\phi$, (c.f. (35) of Corollary 4 and (45) of Theorem 4 below). Essentially, the above loss of derivative has been transferred to the perturbation $\phi$, making it consistent with the use of a Nash-Moser iteration in which the function $\phi$ is mollified at each step of the iteration [3, 7]. The fact that the methods here do not rely on bounds on the difference between nearby shifts, (which is worse than the shifts themselves), makes the methods here that much more interesting, and maybe even a bit surprising. Our hope is that the results established here for the scalar non-uniform difference operator $\mathcal{S}_{\phi}-\mathcal{I}$ will shed light on the 
more complicated multi-component shift operators whose inverse is required to complete the authors' program for establishing periodic solutions of the compressible Euler equations, [7].

The solvability condition (5) is non-local and difficult to verify in general. However, this problem came about as a result of a Nash-Moser iteration, which requires solving the linear equation

$$
D \mathcal{F}_{k}\left(U_{k}\right)\left[V_{k+1}\right]=\mathcal{F}_{k}\left(U_{k}\right)
$$

for $V_{k+1}$, (the Newton iteration step), where $\mathcal{F}_{k}$ is the nonlinear operator and $D \mathcal{F}_{k}\left(U_{k}\right)$ is its linearization around $U_{k}$. In our application, $\mathcal{F}_{k}$ includes nonlinear evolution of a hyperbolic equation, which in turn yields a particular shift operator. It is somewhat surprising that the linearization $D F_{k}\left(U_{k}\right)$ can be expressed in terms of the same shift operator, so that the same shift appears on both sides of the equation, which indicates that the solvability condition may naturally be satisfied in the Nash-Moser iteration.

Finally, it is important to comment on the substantial difficulties encountered by trying to invert $\Delta_{\Phi}=\mathcal{S}_{\Phi}-\mathcal{I}$ by Fourier methods. In fact, we know of no argument employing Fourier analysis sufficient to obtain and estimate the inverse of $\Delta_{\Phi}$. The reason is that the shift operator does not have a nice expression in terms of standard Fourier modes. Now the Fourier framework is most natural for expressing the periodicity conditions in the base linearized problem which has constant coefficients, but under perturbation, the Fourier method encounters shift operators like $\mathcal{S}_{\Phi}$. For example, suppose that $\phi(t)=\sin t$, and consider $\mathcal{S}_{\Phi}$ acting on $L^{2}[0, \pi]$ via $\mathcal{S}_{\Phi} v(t)=v(t+\alpha \sin t)$. Expanding $v$ in Fourier sine series, we can write

$$
v(t)=\sum_{k=0}^{\infty} a_{k} \sin (k t) . \quad \text { so that } \quad \mathcal{S}_{\Phi} v(t)=\sum_{k=0}^{\infty} a_{k} e_{k}(t),
$$

where we have set

$$
e_{k}(t)=\sin (k(t+\alpha \sin t)) .
$$

It is easy to see that $e_{k}(t)$ is an orthonormal basis for $L^{2}[0, \pi]$ with weight function $\sigma=1 /\left(1+\alpha \phi^{\prime}(t)\right)$, so that $\mathcal{S}_{\Phi}$ defines a linear isometry on $L^{2}[0, \pi]$. Our results here show that there are many fixed points of $\mathcal{S}_{\Phi}$ in $L^{2}$, but imposing continuity rules out all but the constants, and we know of no way to establish this via Fourier methods. The problem of inverting $\Delta_{\Phi}$, begins with determining the kernel of $\Delta_{\Phi}$, which reduces to the problem of expressing $e_{k}$ in terms of the standard Fourier basis $\sin (k t)$. This leads to the formula

$$
e_{k}(t)=\sum_{j=0}^{\infty} \int_{0}^{\pi} \sin (j t) \sin (k(t+\alpha \sin t)) d t,
$$

which is problematic because of the essentially random distribution of coefficients in the high modes, even for small $k$. In particular, our results imply that although the change of basis map has many fixed points in $L^{2}$, there 
are no nontrivial continuous fixed points, so none in $H^{1}$. We know of no way to produce this result directly by Fourier methods.

\section{Structure of $\Delta_{\Phi}$}

Recall that we defined the non-uniform difference operator by

$$
\Delta_{\Phi}=\mathcal{S}_{\Phi}-\mathcal{I}, \quad \text { so that } \quad \Delta_{\Phi} v(t)=v(\Phi t)-v(t) .
$$

For $\alpha$ small enough, the shift function $\Phi$ is invertible. We write $\Phi^{k} t$ to denote $k$-fold composition of the shift function $\Phi$, and we let $t_{-\infty}$ and $t_{+\infty}$ denote consecutive zeroes of $\phi$, or equivalently fixed points of $\Phi$. It follows that for any $t$ between $t_{ \pm \infty}$, we have $\Phi^{k} t \rightarrow t_{ \pm \infty}$ as $k \rightarrow \pm \infty$, and indeed $\Phi$ maps the interval $\left[t_{-\infty}, t_{+\infty}\right]$ to itself. Here we are implicitly assuming $\alpha \phi>0$; if not we would have $t_{+\infty}<t_{-\infty}$.

Since $\Phi$ is Lipshitz, it is clear that that $\Delta_{\Phi}$ can be regarded as a map $C^{0,1}\left[t_{-\infty}, t_{+\infty}\right] \rightarrow C^{0,1}\left[t_{-\infty}, t_{+\infty}\right]$. Here $C^{0,1}$ is the space of Lipshitz functions with norm

$$
\|v\|_{C^{0,1}}=\|v\|_{\text {Lip }}+\|v\|_{\text {Sup }},
$$

where $\|v\|_{\text {Sup }}$ denotes the supnorm, and

$$
\|v\|_{L i p}=\sup _{t_{-\infty} \leq t_{a}, t_{b} \leq t_{+\infty}} \frac{\left|v\left(t_{b}\right)-v\left(t_{a}\right)\right|}{\left|t_{b}-t_{a}\right|} .
$$

If $v \in C^{0,1}\left[t_{-\infty}, t_{+\infty}\right]$, the following estimate follows immediately:

$$
\left\|\Delta_{\Phi} v(t)\right\|_{\text {Lip }} \leq\left(1+\|\Phi\|_{\text {Lip }}\right)\|v\|_{\text {Lip }} .
$$

That is, $\Delta_{\Phi}$ is a bounded linear map from $C^{0,1}$ to itself, and our goal here is to examine the extent to which it is invertible. To do this we first identify the kernel and range of $\Delta_{\Phi}$.

2.1. The Kernel. We first characterize the kernel of $\Delta_{\Phi}=\mathcal{S}_{\Phi}-\mathcal{I}$ in the space $L^{\infty}\left[t_{-\infty}, t_{+\infty}\right]$, and then show that the only continuous functions in the kernel are the constant functions.

First, choose any $t_{0} \in\left(t_{-\infty}, t_{+\infty}\right)$, and note that we can write the interval as a disjoint union,

$$
\left(t_{-\infty}, t_{+\infty}\right)=\bigcup_{k}\left[\Phi^{k} t_{0}, \Phi^{k+1} t_{0}\right)
$$

It follows that if

$$
v \in \operatorname{ker} \Delta_{\Phi}, \quad \text { so that } \quad v(\Phi t)=v(t),
$$

then for any $t$ and any $k$, we have

$$
v\left(\Phi^{k} t\right)=v(t) .
$$

For fixed $t_{0}$, any $\tilde{v}_{0} \in L^{\infty}\left[t_{0}, \Phi t_{0}\right)$ thus determines an $L^{\infty}$ element of the kernel, via

$$
v(t)=\tilde{v}_{0}\left(\Phi^{k} t\right), \quad k \text { such that } \Phi^{k} t \in\left[t_{0}, \Phi t_{0}\right)
$$


and every $L^{\infty}$ element of the kernel of $\Delta_{\Phi}$ must come from some such $\tilde{v}_{0}$. Thus (10) puts the kernel of $\Delta_{\Phi}$ in $1-1$ correspondence with $L^{\infty}\left[t_{0}, \Phi t_{0}\right.$ ), and the resulting solutions are defined independent of what base point $t_{0}$ is chosen.

Because $\Phi^{k} t \rightarrow t_{ \pm \infty}$ as $k \rightarrow \pm \infty$, all the values of $\tilde{v}_{0}$ occur in any neighborhood of $t_{ \pm \infty}$ for large enough $k$, so that elements of the kernel will become discontinuous at $t_{ \pm \infty}$ unless $\tilde{v}_{0}(t)=V_{0}=$ Const for all $t \in\left[t_{0}, \Phi t_{0}\right)$. We state this as a lemma:

Lemma 1. Let $t_{0} \in\left(t_{-\infty}, t_{+\infty}\right)$. Then the kernel of $\Delta_{\Phi}$ in $L^{\infty}\left(t_{-\infty}, t_{+\infty}\right)$ is characterized as the set of all functions $\tilde{v}_{0} \in L^{\infty}\left[t_{0}, \Phi t_{0}\right)$ extended to all of $L^{\infty}\left(t_{-\infty}, t_{+\infty}\right)$ by the shift condition (10). Moreover, the only continuous solutions that lie in the $L^{\infty}$ kernel of $\Delta_{\Phi}$ are constants, $v(t)=V_{0}$.

Since the space of Lipshitz continuous functions $C^{0,1}$ contains only continuous functions, we have the following corollary:

Corollary 1. The only elements of the kernel of the difference operator $\Delta_{\Phi}$ in the space $C^{0,1}\left[t_{-\infty}, t_{+\infty}\right]$ are the constant functions $v(t)=V_{0}$.

2.2. The Range of $\Delta_{\Phi}$. We next obtain a condition on $w$ to be in the range of $\Delta_{\Phi}$ for continuous inputs $v \in C\left[t_{-\infty}, t_{+\infty}\right]$. Thus write

$$
w=\Delta_{\Phi} v=\mathcal{S}_{\Phi} v-v .
$$

We solve directly for $v$ in terms of $w$. To do so, write

$$
\begin{aligned}
v(\Phi t) & =v(t)+w(t) \\
v\left(\Phi^{2} t\right) & =v(\Phi t)+w(\Phi t) \\
\vdots & \\
v\left(\Phi^{k} t\right) & =v\left(\Phi^{k-1} t\right)+w\left(\Phi^{k-1} t\right),
\end{aligned}
$$

and combine these to obtain

$$
v(t)=v\left(\Phi^{k} t\right)-\sum_{j=0}^{k-1} w\left(\Phi^{j} t\right),
$$

for $k>0$. Replacing $t$ by $\Phi^{-k} t$, we also get

$$
v(t)=v\left(\Phi^{-k} t\right)+\sum_{l=1}^{k} w\left(\Phi^{-l} t\right) .
$$

Now taking the limit $k \rightarrow \infty$ in these gives

$$
\begin{aligned}
& v(t)=v\left(t_{+\infty}\right)-\sum_{j=0}^{\infty} w\left(\Phi^{j} t\right), \quad \text { and } \\
& v(t)=v\left(t_{-\infty}\right)+\sum_{l=1}^{\infty} w\left(\Phi^{-l} t\right),
\end{aligned}
$$


since $v$ is continuous at $t_{ \pm \infty}$. Equating these yields a condition on any function $w$ in the range of $\Delta_{\Phi}$ with continuous input, namely

$$
\sum_{k=-\infty}^{+\infty} w\left(\Phi^{k} t\right)=\text { Const }=v\left(t_{+\infty}\right)-v\left(t_{-\infty}\right)
$$

Since $w(t)=v(\Phi t)-v(t), w$ is continuous when $v$ is continuous, so, by construction, we have proven the following lemma which characterizes the range of $\Delta_{\Phi}$ :

Lemma 2. The range of the operator $\Delta_{\Phi} v=\mathcal{S}_{\Phi}-\mathcal{I}$ on $C\left[t_{-\infty}, t_{+\infty}\right]$ is the set of all $w \in C\left[t_{-\infty}, t_{+\infty}\right]$ such that the infinite sums converge for all $t$,

$$
\left|\sum_{j=0}^{\infty} w\left(\Phi^{j} t\right)\right|<\infty, \quad\left|\sum_{k=1}^{\infty} w\left(\Phi^{-k} t\right)\right|<\infty,
$$

and such that (12) holds, namely

$$
\sum_{k=-\infty}^{+\infty} w\left(\Phi^{k} t\right)=\text { Const }
$$

In this case, either equation in (11) defines $v(t)$ such that $\Delta_{\Phi} v=w$ to within a constant.

\section{INVERTIBILITY OF $\Delta_{\Phi}$}

Our goal now is to prove that the difference operator $\Delta_{\Phi}$ is invertible on its range; that is, given a Lipshitz function $w$ satisfying (13) and (12), we construct a function $v$ satisfying $\Delta_{\Phi} v=w$, and derive appropriate bounds on the solution $v$. We know from (11) how the solution $v$ must be defined, and the main issue is to show that (12) is a sufficient condition for solvability, and then to obtain bounds on $v$ in terms of $w$. We begin by examining the iterations of the shift function in more detail.

3.1. Properties of the shift. Recall that the shift function is defined by (2), namely

$$
\Phi(t)=t+\alpha \phi(t)
$$

where we assume $\phi$ is non-degenerate, so that (2)-(3) hold. For definiteness, assume that $\alpha \phi(t)>0$ on the interval $\left(t_{-\infty}, t_{+\infty}\right)$, which in turn implies $\phi^{\prime}\left(t_{+\infty}\right)<0<\phi^{\prime}\left(t_{-\infty}\right)$; similar statements and estimates hold if $\alpha \phi(t)<0$ and $t_{+\infty}<t_{-\infty}$. We begin by bounding $\phi(t)$ away from zero by a trapezoid, and use this to show that for any $\alpha \neq 0$, a finite number of iterations takes us from a neighborhood of $t_{-\infty}$ into a neighborhood of $t_{+\infty}$.

From this point on in the paper, assume without loss of generality that $\phi(t) \geq 0$, so that $\phi^{\prime}\left(t_{-\infty}\right)>0, \phi^{\prime}\left(t_{+\infty}\right)<0$, and that $\alpha>0$, so $t_{-\infty}<t_{+\infty}$. All proofs carry over to the case $\alpha \phi^{\prime}\left(t_{-\infty}\right)>0, \alpha \phi^{\prime}\left(t_{+\infty}\right)<0$ with slight modification. 


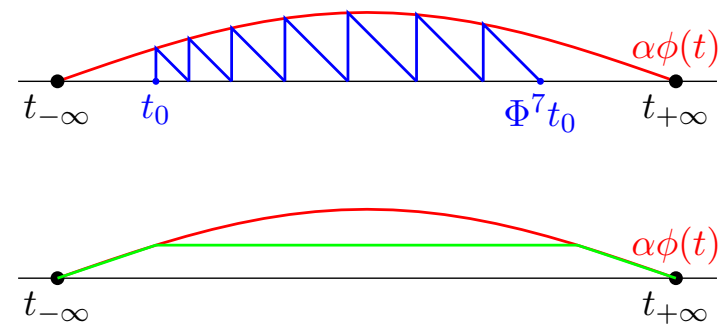

FiguRE 1. The Shift Function

Lemma 3. There are Lipshitz functions $E_{+}(t)$ and $E_{-}(t)$, vanishing at $t_{+\infty}$ and $t_{-\infty}$, respectively, such that, for both \pm we have,

$$
\phi(t)=\phi^{\prime}\left(t_{ \pm \infty}\right)\left\{1+E_{ \pm}(t)\right\}\left(t-t_{ \pm \infty}\right) .
$$

In particular, given any $\delta>0$, there are $\epsilon_{\phi}>0$ and $m_{\phi}>0$ such that

$$
\begin{array}{lll}
\left|E_{-}(t)\right|<\delta & \text { for } \quad t_{-\infty} \leq t \leq t_{-\infty}+\epsilon_{\phi}, \quad \text { and } \\
\left|E_{+}(t)\right|<\delta & \text { for } \quad t_{+\infty}-\epsilon_{\phi} \leq t \leq t_{+\infty},
\end{array}
$$

and

$$
|\phi(t)| \geq m_{\phi} \quad \text { for } \quad t_{-\infty}+\epsilon_{\phi} \leq t \leq t_{+\infty}-\epsilon_{\phi} .
$$

Proof. Undoing (14), for $t \neq t_{ \pm \infty}$, define the functions $E_{ \pm}$by

$$
\begin{aligned}
E_{ \pm}(t) & :=\frac{\phi(t)}{\phi^{\prime}\left(t_{ \pm \infty}\right)\left(t-t_{ \pm \infty}\right)}-1 \\
& =\frac{\phi(t)-\phi\left(t_{ \pm \infty}\right)-\phi^{\prime}\left(t_{ \pm \infty}\right)\left(t-t_{ \pm \infty}\right)}{\phi^{\prime}\left(t_{ \pm \infty}\right)\left(t-t_{ \pm \infty}\right)} .
\end{aligned}
$$

Since $\phi$ is Lipshitz, $E_{ \pm}$are Lipshitz away from $t_{ \pm \infty}$, respectively, so we must show boundedness and continuity at $t_{ \pm \infty}$. It follows directly from (3) and (44) that $E_{ \pm}$can be extended to Lipshitz functions on all of $\left[t_{-\infty}, t_{+\infty}\right]$, and that $E_{ \pm}\left(t_{ \pm \infty}\right)=0$.

Now if $\delta>0$ is given, let $E_{\phi}$ be a Lipshitz bound for $E_{ \pm}$,

$$
\left\|E_{ \pm}\right\|_{L i p} \leq E_{\phi}, \quad \text { and take } \quad \epsilon_{\phi}<\frac{\delta}{E_{\phi}}
$$

so that (15) holds.

Finally, we can choose $m_{\phi}>0$ because $\phi(t)$ is non-zero on the compact interval $\left[t_{-\infty}+\epsilon_{\phi}, t_{+\infty}-\epsilon_{\phi}\right]$. Define

$$
\phi_{\infty}^{\prime}=\min \left\{\left|\phi^{\prime}\left(t_{-\infty}\right)\right|,\left|\phi^{\prime}\left(t_{+\infty}\right)\right|\right\},
$$

and restrict the size of $\epsilon_{\phi}$ if necessary, to obtain

$$
m_{\phi}=\phi_{\infty}^{\prime} \epsilon_{\phi}(1-\delta)
$$


Then $\phi(t)$ is bounded below by the trapezoid,

$$
\phi(t) \geq \begin{cases}\phi_{\infty}^{\prime}(1-\delta)\left(t-t_{-\infty}\right), & t_{-\infty} \leq t \leq t_{-\infty}+\epsilon_{\phi}, \\ \phi_{\infty}^{\prime}(1-\delta) \epsilon_{\phi} & t_{-\infty}+\epsilon_{\phi} \leq t \leq t_{+\infty}-\epsilon_{\phi}, \\ \phi_{\infty}^{\prime}(1-\delta)\left(t_{+\infty}-t\right), & t_{+\infty}+\epsilon_{\phi} \leq t \leq t_{+\infty}\end{cases}
$$

as pictured in Figure 1

Recall that $\Phi$ given by (2) is invertible for all $\alpha$ satisfying

$$
|\alpha|<\alpha_{\phi}:=\frac{1}{\|\phi\|_{\text {Lip }}} .
$$

For convenience, assume now, without loss of generality, that

$$
\epsilon_{\phi}<\left|t_{+\infty}-t_{-\infty}\right| / 4 \text {. }
$$

Corollary 2. For any non-degenerate $\phi$ and $\alpha$ satisfying (19), there is a positive integer $N_{\alpha}$ depending only on $\alpha$ and $m_{\phi}$, such that for any $k \geq N_{\alpha}$ and $t \in\left[t_{-\infty}+\epsilon_{\phi}, t_{+\infty}-\epsilon_{\phi}\right]$, we have both

$$
\Phi^{-k} t \in\left(t_{-\infty}, t_{-\infty+\epsilon_{\phi}}\right) \quad \text { and } \Phi^{k} t \in\left(t_{+\infty}-\epsilon_{\phi}, t_{+\infty}\right) .
$$

Proof. For definiteness, suppose that $\alpha \phi(t)>0$. As long as $t$ and $\Phi^{j} t$ remain in the interval $\left[t_{-\infty}+\epsilon_{\phi}, t_{+\infty}-\epsilon_{\phi}\right]$, we have

$$
\Phi t \geq t+\alpha m_{\phi}, \quad \text { and } \quad \Phi^{j} t \geq t+j \alpha m_{\phi},
$$

so it suffices to take

$$
N_{\alpha} \geq \frac{t_{+\infty}-\epsilon_{\phi}-\left(t_{-\infty}+\epsilon_{\phi}\right)}{\alpha m_{\phi}}+1
$$

Note that if $\alpha \phi<0$, the intervals change: in that case

$$
\Phi^{-k} t \in\left(t_{-\infty}-\epsilon_{\phi}, t_{-\infty}\right) \text { and } \Phi^{k} t \in\left(t_{+\infty}, t_{+\infty}+\epsilon_{\phi}\right),
$$

for $t \in\left[t_{+\infty}+\epsilon_{\phi}, t_{-\infty}-\epsilon_{\phi}\right]$.

We now show that because $\phi$ is non-degenerate, iteration of the shift map $\Phi$ gives a geometric progression into the fixed points, so we can use a geometric series to control the cumulative iterations.

Theorem 1. Let $\delta>0$ be given and let $\epsilon_{\phi}, m_{\phi}$ and $N_{\alpha}$ be determined so that Lemma 3 and Corollary 2 hold, and assume $t_{a} \leq t_{b}$. If $t_{a} \geq t_{-\infty}+\epsilon_{\phi}$, then

$$
\sum_{k=0}^{\infty}\left|\Phi^{k} t_{b}-\Phi^{k} t_{a}\right| \leq V_{\phi}\left|t_{b}-t_{a}\right|,
$$

and if $t_{b} \leq t_{+\infty}-\epsilon_{\phi}$, then

$$
\sum_{k=0}^{-\infty}\left|\Phi^{k} t_{b}-\Phi^{k} t_{a}\right| \leq V_{\phi}\left|t_{b}-t_{a}\right|,
$$

where

$$
V_{\phi}=\frac{2\left(1+|\alpha|\|\phi\|_{L i p}\right)^{N_{\alpha}}}{|\alpha| \phi_{\infty}^{\prime}(1-2 \delta)} .
$$


Proof. For definiteness, assume that $t_{a} \geq t_{-\infty}+\epsilon_{\phi}$. We prove (21); a similar argument yields (22) when $t_{b} \leq t_{+\infty}-\epsilon_{\phi}$. We break the proof into two cases:

(i) $t_{a} \geq t_{+\infty}-\epsilon_{\phi}$; and

(ii) $t_{a} \in\left(t_{-\infty}+\epsilon_{\phi}, t_{+\infty}-\epsilon_{\phi}\right)$.

Consider first case (i). Then also $t_{b} \geq t_{+\infty}-\epsilon_{\phi}$, and we can use (14) to write

$$
\begin{aligned}
& \phi\left(t_{b}\right)=\phi^{\prime}\left(t_{+\infty}\right)\left\{1+E_{+}\left(t_{b}\right)\right\}\left(t_{b}-t_{+\infty}\right), \\
& \phi\left(t_{a}\right)=\phi^{\prime}\left(t_{+\infty}\right)\left\{1+E_{+}\left(t_{a}\right)\right\}\left(t_{a}-t_{+\infty}\right),
\end{aligned}
$$

and recall we have assumed $\phi>0$ so that $\phi^{\prime}\left(t_{+\infty}\right)<0$. Subtracting and rearranging, we obtain

$$
\begin{aligned}
\phi\left(t_{b}\right) & -\phi\left(t_{a}\right) \\
& =\phi^{\prime}\left(t_{+\infty}\right)\left\{t_{b}-t_{a}+E_{+}\left(t_{b}\right)\left(t_{b}-t_{+\infty}\right)-E_{+}\left(t_{a}\right)\left(t_{a}-t_{+\infty}\right)\right\} \\
& =\phi^{\prime}\left(t_{+\infty}\right)\left\{t_{b}-t_{a}+E_{+}\left(t_{b}\right)\left(t_{b}-t_{a}\right)+\left(E_{+}\left(t_{b}\right)-E_{+}\left(t_{a}\right)\right)\left(t_{a}-t_{+\infty}\right)\right\} \\
& =\phi^{\prime}\left(t_{+\infty}\right)\left\{1+E_{+}\left(t_{b}\right)+\frac{E_{+}\left(t_{b}\right)-E_{+}\left(t_{a}\right)}{t_{b}-t_{a}}\left(t_{a}-t_{+\infty}\right)\right\}\left(t_{b}-t_{a}\right) .
\end{aligned}
$$

Now, according to (15), we have $\left|E_{+}\left(t_{b}\right)\right| \leq \delta$, and by (16), we have

$$
\left|\frac{E_{+}\left(t_{b}\right)-E_{+}\left(t_{a}\right)}{t_{b}-t_{a}}\left(t_{b}-t_{+\infty}\right)\right| \leq E_{\phi} \epsilon_{\phi} \leq \delta .
$$

Thus, since $\phi^{\prime}\left(t_{+\infty}\right)<0$, we can estimate

$$
\left.\left.\phi^{\prime}\left(t_{+\infty}\right)\{1+2 \delta)\right\}\left(t_{b}-t_{a}\right) \leq \phi\left(t_{b}\right)-\phi\left(t_{a}\right) \leq \phi^{\prime}\left(t_{+\infty}\right)\{1-2 \delta)\right\}\left(t_{b}-t_{a}\right)
$$

which in turn gives

$$
\left|\Phi t_{b}-\Phi t_{a}\right| \leq\left\{1-\left|\alpha \phi^{\prime}\left(t_{+\infty}\right)\right|(1-2 \delta)\right\}\left(t_{b}-t_{a}\right) .
$$

By induction conclude that

$$
\left|\Phi^{k} t_{b}-\Phi^{k} t_{a}\right| \leq\left\{1-\left|\alpha \phi^{\prime}\left(t_{+\infty}\right)\right|(1-2 \delta)\right\}^{k}\left(t_{b}-t_{a}\right),
$$

and thus

$$
\begin{aligned}
\sum_{k=0}^{\infty}\left|\Phi^{k} t_{b}-\Phi^{k} t_{a}\right| & \leq \sum_{k=0}^{\infty}\left\{1-\left|\alpha \phi^{\prime}\left(t_{+\infty}\right)\right|(1-2 \delta)\right\}^{k}\left(t_{b}-t_{a}\right) \\
& \leq V_{1}\left(t_{b}-t_{a}\right)
\end{aligned}
$$

where we have set

$$
V_{1}:=\frac{1}{\left|\alpha \phi_{\infty}^{\prime}\right|(1-2 \delta)}
$$

this completes the proof in case (i). 
Consider now case (ii). Since $t_{-\infty}+\epsilon_{\phi} \leq t_{a} \leq t_{b}$, by Corollary 2 we know that $t_{+\infty}-\epsilon_{\phi} \leq \Phi^{k} t_{a}<\Phi^{k} t_{b}$ for all $k \geq N_{\alpha}$, and we write

$$
\sum_{k=0}^{\infty}\left|\Phi^{k} t_{b}-\Phi^{k} t_{a}\right|=\sum_{k=0}^{N_{\alpha}-1}\left|\Phi^{k} t_{b}-\Phi^{k} t_{a}\right|+\sum_{k=N_{\alpha}}^{\infty}\left|\Phi^{k} t_{b}-\Phi^{k} t_{a}\right| .
$$

Since all the terms in the second sum lie within $\epsilon_{\phi}$ of $t_{+\infty}$, we can estimate the second sum by case (i) and obtain

$$
\sum_{k=N_{\alpha}}^{\infty}\left|\Phi^{k} t_{b}-\Phi^{k} t_{a}\right| \leq V_{1}\left|\Phi^{N_{\alpha}} t_{b}-\Phi^{N_{\alpha}} t_{a}\right|
$$

Now $\left|\Phi t_{2}-\Phi t_{1}\right| \leq\left(1+|\alpha|\|\phi\|_{L i p}\right)\left|t_{2}-t_{1}\right|$, so by induction,

$$
\left|\Phi^{k} t_{2}-\Phi^{k} t_{1}\right| \leq\left(1+|\alpha|\|\phi\|_{L i p}\right)^{k}\left|t_{2}-t_{1}\right| .
$$

Thus, the second sum in (25) is bounded by

$$
\sum_{k=N_{\alpha}}^{\infty}\left|\Phi^{k} t_{b}-\Phi^{k} t_{a}\right| \leq V_{1}\left(1+|\alpha|\|\phi\|_{L i p}\right)^{N_{\alpha}}\left|t_{b}-t_{a}\right| .
$$

Consider next the first sum in (25). By (26) we can estimate

$$
\begin{aligned}
\sum_{k=0}^{N_{\alpha}-1}\left|\Phi^{k} t_{b}-\Phi^{k} t_{a}\right| & \leq \sum_{k=0}^{N_{\alpha}-1}\left(1+|\alpha|\|\phi\|_{\text {Lip }}\right)^{k}\left(t_{b}-t_{a}\right) \\
& \leq \frac{\left(1+|\alpha|\|\phi\|_{\text {Lip }}\right)^{N_{\alpha}}-1}{|\alpha|\|\phi\|_{\text {Lip }}}\left|t_{b}-t_{a}\right|,
\end{aligned}
$$

where we have summed the finite geometric series.

Combining the estimates for the sums in (25), we get

$$
\sum_{k=0}^{\infty}\left|\Phi^{k} t_{b}-\Phi^{k} t_{a}\right| \leq V_{\phi}\left|t_{b}-t_{a}\right|
$$

where we have written

$$
\begin{gathered}
V_{1}\left(1+|\alpha|\|\phi\|_{L i p}\right)^{N_{\alpha}}+\frac{\left(1+|\alpha|\|\phi\|_{\text {Lip }}\right)^{N_{\alpha}}-1}{|\alpha|\|\phi\|_{\text {Lip }}} \\
\leq \frac{2\left(1+|\alpha|\|\phi\|_{\text {Lip }}\right)^{N_{\alpha}}}{|\alpha| \phi_{\infty}^{\prime}(1-2 \delta)}=: V_{\phi},
\end{gathered}
$$

since $\phi_{\infty}^{\prime} \leq\|\phi\|_{\text {Lip }}$. This establishes case (ii), and completes the proof of the theorem.

3.2. Convergence of the infinite sums. Our goal is to prove invertibility of $\Delta_{\Phi}$, which amounts to proving consistency of and estimates for our construction (11), which expresses the solution $v$ of the equation

$$
\Delta_{\Phi} v=\mathcal{S}_{\Phi} v-v=w
$$

as an infinite series. We begin by using the geometric convergence of iterations of $\Phi$ to simplify the condition for convergence of these series. 
Lemma 4. Assume that the conditions of Theorem 1 holds, and let $w$ be Lipschitz continuous on $\left[t_{-\infty}, t_{+\infty}\right]$. Then the series in (13) converge if and only if $w$ vanishes at the fixed points,

$$
w\left(t_{-\infty}\right)=0 \quad \text { and } \quad w\left(t_{+\infty}\right)=0 .
$$

Moreover, if $t$ lies within $\epsilon_{\phi}$ of one of the endpoints $t_{ \pm \infty}$, we have the bound

$$
\left|\sum_{k=0}^{\infty} w\left(\Phi^{ \pm k} t\right)\right| \leq\|w\|_{L i p} \frac{\left|t_{ \pm \infty}-t\right|}{|\alpha|(1-\delta) \phi_{\infty}^{\prime}},
$$

where $\phi_{\infty}^{\prime}$ is given by (17).

Proof. Assuming convergence of the series (13),

$$
\left|\sum_{k=0}^{\infty} w\left(\Phi^{k} t\right)\right|<\infty \text { and }\left|\sum_{k=0}^{\infty} w\left(\Phi^{-k} t\right)\right|<\infty,
$$

it follows that $w\left(\Phi^{ \pm k} t\right) \rightarrow 0$, and so by continuity,

$$
w\left(t_{ \pm \infty}\right)=w\left(\lim _{k \rightarrow \infty} \Phi^{ \pm k} t\right)=\lim _{k \rightarrow \infty} w\left(\Phi^{ \pm k} t\right)=0 .
$$

For the reverse implication, assume that $w\left(t_{+\infty}\right)=0$. We show the first sum in (13) is finite; the other case is similar. Since only finitely many terms in each sum of (13) lie $\epsilon_{\phi}$ away from $t_{ \pm \infty}$, it suffices to prove

$$
\left|\sum_{k=0}^{\infty} w\left(\Phi^{k} t\right)\right|<\infty \quad \text { for } \quad t \in\left(t_{+\infty}-\epsilon_{\phi}, t_{+\infty}\right) .
$$

We write $t_{0}:=t>t_{+\infty}-\epsilon_{\phi}$ and set $t_{k}=\Phi^{k} t$. Using (21) and (14), we write

$$
t_{k+1}=t_{k}+\alpha \phi^{\prime}\left(t_{+\infty}\right)\left\{1+E_{+}\left(t_{k}\right)\right\}\left(t_{k}-t_{+\infty}\right),
$$

which in turn yields

$$
\begin{aligned}
t_{+\infty}-t_{k+1} & =\left(1+\alpha \phi^{\prime}\left(t_{+\infty}\right)\left\{1+E_{+}\left(t_{k}\right)\right\}\right)\left(t_{+\infty}-t_{k}\right) \\
& \leq\left(1-\left|\alpha \phi^{\prime}\left(t_{+\infty}\right)\right|(1-\delta)\right)\left(t_{+\infty}-t_{k}\right),
\end{aligned}
$$

where we have used (15), and recalled that $\alpha \phi^{\prime}\left(t_{+\infty}\right)<0$. Continuing by induction we obtain

$$
t_{\infty}-t_{k} \leq\left(1-\left|\alpha \phi^{\prime}\left(t_{+\infty}\right)\right|(1-\delta)\right)^{k}\left(t_{+\infty}-t\right),
$$

and since $w\left(t_{+\infty}\right)=0$, we can write

$$
\begin{aligned}
\left|\sum_{k=0}^{+\infty} w\left(\Phi^{k} t\right)\right| & \leq \sum_{k=0}^{\infty}\left|w\left(t_{+\infty}\right)-w\left(\Phi^{k} t\right)\right| \leq \sum_{k=0}^{+\infty}\|w\|_{\text {Lip }}\left|t_{+\infty}-t_{k}\right| \\
& \leq\|w\|_{\text {Lip }} \sum_{k=0}^{+\infty}\left(1-\left|\alpha \phi^{\prime}\left(t_{+\infty}\right)\right|(1-\delta)\right)^{k}\left(t_{+\infty}-t\right) \\
& \leq\|w\|_{\text {Lip }} \frac{t_{+\infty}-t}{\left|\alpha \phi^{\prime}\left(t_{+\infty}\right)\right|(1-\delta)}<\infty
\end{aligned}
$$


The argument in the symmetrical case $t \in\left(t_{-\infty}, t_{-\infty}+\epsilon_{\phi}\right)$ gives the result

$$
\left|\sum_{k=0}^{-\infty} w\left(\Phi^{-k} t\right)\right| \leq\|w\|_{L i p} \frac{t-t_{-\infty}}{\left|\alpha \phi^{\prime}\left(t_{-\infty}\right)\right|(1-\delta)},
$$

and these together yield (27).

As a corollary, we obtain bounds on the full sum $\sum_{k=-\infty}^{\infty} w\left(\Phi^{k} t\right)$ provided $w$ vanishes at $t_{ \pm \infty}$.

Corollary 3. Assume $w$ is Lipschitz continuous and satisfies $w\left(t_{ \pm \infty}\right)=0$, and the conditions of Theorem 1 hold. Then for any $t \in\left[t_{-\infty}, t_{+\infty}\right]$,

$$
\left|\sum_{k=-\infty}^{\infty} w\left(\Phi^{k} t\right)\right| \leq\|w\|_{\text {Lip }}\left\{\frac{2}{|\alpha|(1-\delta) \phi_{\infty}^{\prime}}+N_{\alpha}\right\}\left|t_{+\infty}-t_{-\infty}\right| .
$$

Proof. Since the $\Phi^{k} t \rightarrow t_{ \pm \infty}$ as $k \rightarrow \pm \infty$, we can replace $t$ by $\Phi^{j} t$ for any convenient integer $j$. Using Corollary 2, we choose $t_{0}=\Phi^{j} t$ such that

$$
t_{0} \leq t_{-\infty}+\epsilon_{\phi} \text { and } \Phi^{N_{\alpha}+1} t_{0} \geq t_{+\infty}-\epsilon_{\phi} .
$$

Then we have

$$
\left|\sum_{k=-\infty}^{\infty} w\left(\Phi^{k} t\right)\right| \leq\left|\sum_{k=0}^{-\infty} w\left(\Phi^{-k} t_{0}\right)\right|+\left|\sum_{k=1}^{N_{\alpha}} w\left(\Phi^{k} t_{0}\right)\right|+\left|\sum_{k=N_{\alpha}+1}^{\infty} w\left(\Phi^{k} t_{0}\right)\right|,
$$

and the first and third sums are estimated by (27). We estimate the middle term by

$$
\left|\sum_{k=1}^{N_{\alpha}} w\left(\Phi^{k} t_{0}\right)\right| \leq N_{\alpha}\|w\|_{S u p} \leq N_{\alpha}\|w\|_{L i p}\left|t_{+\infty}-t_{-\infty}\right|
$$

since $w$ vanishes at $t_{ \pm \infty}$. Combining the three terms yields (28).

3.3. Invertibility in the space $C^{0,1}$. We now state and prove our main theorem, which states that the operator $\Delta_{\Phi}=\mathcal{S}_{\Phi}-\mathcal{I}$ is invertible on its range, and that the inverse is bounded in the $C^{0,1}$ norm.

Theorem 2. Assume that $\phi(t)$ is non-degenerate, let $\delta>0$ and

$$
|\alpha|<\frac{1-\delta}{\phi_{\infty}^{\prime}}<\frac{1}{\|\phi\|_{\text {Lip }}}
$$

be given, and choose $\epsilon_{\phi}<\left(t_{+\infty}-t_{-\infty}\right) / 4$ and $N_{\alpha}$ so that the conditions of Theorem 1 hold. Also let $w \in C^{0,1}\left[t_{-\infty}, t_{+\infty}\right]$ satisfy $w\left(t_{-\infty}\right)=w\left(t_{+\infty}\right)=0$ and the consistency condition (12), namely

$$
\sum_{k=-\infty}^{+\infty} w\left(\Phi^{k} t\right)=\text { Const. }
$$

Then the equation

$$
\Delta_{\Phi} v(t)=v(\Phi t)-v(t)=w(t)
$$


has a solution $v \in C^{0,1}\left[t_{-\infty}, t_{+\infty}\right]$, uniquely determined up to constant, which is explicitly given by either equation in (11), which are consistent. Moreover, the solution satisfies the bound

$$
\|v\|_{\text {Lip }} \leq K_{\phi}\|w\|_{\text {Lip }}, \quad \text { so that } \quad\left\|\Delta_{\Phi}^{-1}\right\| \leq K_{\phi}
$$

where $K_{\phi}$ is given by

$$
K_{\phi}=\max \left\{K_{1}, V_{\phi}\right\}
$$

where

$$
K_{1}=\frac{2+2 \epsilon_{\phi}+|\alpha| N_{\alpha}(1-\delta) \phi_{\infty}^{\prime}}{|\alpha|(1-\delta) \phi_{\infty}^{\prime}\left(t_{+\infty}-t_{-\infty}-2 \epsilon_{\phi}\right)}
$$

and $V_{\phi}$ is given in (23).

Proof. According to Lemma 4, the conditions $w\left(t_{ \pm \infty}\right)=0$ imply that the infinite series converge, so that equations (11) make sense. We can choose one of $v\left(t_{ \pm \infty}\right)$ arbitrarily, and the other is determined by (12), which also implies consistency of both equations in (11). Since the solution is given by an explicit formula, it is unique up to our choice of constant, and all that remains is to establish (29).

As above, for definiteness we assume that $\alpha \phi(t)>0$; similar estimates hold for the other case. Assume that we are given $t_{a}$ and $t_{b} \in\left[t_{-\infty}, t_{+\infty}\right]$, and suppose $t_{a}<t_{b}$. We again consider two cases:

(i) $t_{a} \geq t_{-\infty}+\epsilon_{\phi}$ or $t_{b} \leq t_{+\infty}-\epsilon_{\phi}$; and

(ii) $t_{a}<t_{-\infty}+\epsilon_{\phi}$ and $t_{b}>t_{+\infty}-\epsilon_{\phi}$.

For the first case, we assume $t_{a} \geq t_{-\infty}+\epsilon_{\phi}$; the case $t_{b} \leq t_{+\infty}-\epsilon_{\phi}$ follows similarly. Here Theorem 11 applies directly: we use the first equation in (11) to describe $v(t)$ and write

$$
\begin{aligned}
\left|v\left(t_{b}\right)-v\left(t_{a}\right)\right| & =\left|\sum_{k=0}^{\infty}\left(w\left(\Phi^{k} t_{b}\right)-w\left(\Phi^{k} t_{a}\right)\right)\right| \\
& \leq\|w\|_{\text {Lip }} \sum_{k=0}^{\infty}\left|\Phi^{k} t_{b}-\Phi^{k} t_{a}\right| \\
& \leq\|w\|_{\text {Lip }} V_{\phi}\left|t_{b}-t_{a}\right|,
\end{aligned}
$$

where we have applied (21). When $t_{b} \leq t_{+\infty}-\epsilon_{\phi}$, the same estimate holds using the second equation of (11) and (22). This is the required estimate for case (i).

We now consider case (ii), in which $t_{a}$ and $t_{b}$ lie within $\epsilon_{\phi}$ of different fixed points. We use different expressions for $v\left(t_{a}\right)$ and $v\left(t_{b}\right)$; by (11), we have

$$
\begin{aligned}
& v\left(t_{a}\right)=v\left(t_{-\infty}\right)+\sum_{k=1}^{\infty} w\left(\Phi^{-k} t_{a}\right), \quad \text { and } \\
& v\left(t_{b}\right)=v\left(t_{+\infty}\right)-\sum_{k=0}^{\infty} w\left(\Phi^{k} t_{b}\right)
\end{aligned}
$$


Subtracting, taking the absolute value, and applying (27) gives

$$
\left|v\left(t_{b}\right)-v\left(t_{b}\right)\right| \leq\left|v\left(t_{+\infty}\right)-v\left(t_{-\infty}\right)\right|+\|w\|_{L i p} \frac{2 \epsilon_{\phi}}{|\alpha|(1-\delta) \phi_{\infty}^{\prime}} .
$$

We use (28) to write

$$
\left|v\left(t_{+\infty}\right)-v\left(t_{-\infty}\right)\right| \leq\|w\|_{\text {Lip }}\left\{\frac{2}{|\alpha|(1-\delta) \phi_{\infty}^{\prime}}+N_{\alpha}\right\}\left|t_{+\infty}-t_{-\infty}\right|,
$$

so that (33) becomes

$$
\left|v\left(t_{b}\right)-v\left(t_{b}\right)\right| \leq\|w\|_{L i p} K_{0}
$$

where we have set

$$
\begin{aligned}
K_{0} & :=\left\{\frac{2}{|\alpha|(1-\delta) \phi_{\infty}^{\prime}}+N_{\alpha}\right\}\left|t_{+\infty}-t_{-\infty}\right|+\frac{2 \epsilon_{\phi}}{|\alpha|(1-\delta) \phi_{\infty}^{\prime}} \\
& =\frac{2+2 \epsilon_{\phi}+|\alpha| N_{\alpha}(1-\delta) \phi_{\infty}^{\prime}}{|\alpha|(1-\delta) \phi_{\infty}^{\prime}} .
\end{aligned}
$$

Finally, since

$$
t_{b}-t_{a}>t_{+\infty}-t_{-\infty}-2 \epsilon_{\phi}
$$

we can write

$$
\left|v\left(t_{b}\right)-v\left(t_{a}\right)\right| \leq\|w\|_{L i p} K_{1}\left|t_{b}-t_{a}\right|
$$

where we have set

$$
K_{1}:=\frac{K_{0}}{t_{+\infty}-t_{-\infty}-2 \epsilon_{\phi}}=\frac{2+2 \epsilon_{\phi}+|\alpha| N_{\alpha}(1-\delta) \phi_{\infty}^{\prime}}{|\alpha|(1-\delta) \phi_{\infty}^{\prime}\left(t_{+\infty}-t_{-\infty}-2 \epsilon_{\phi}\right)},
$$

which is (31). Combining (32) and (34) yields (29), and the proof is complete.

Corollary 4. Since the dependence of $K_{\phi}$ in (31) is given by

$$
K_{\phi} \equiv K_{\phi}\left(\alpha, \delta, \epsilon_{\phi}, N_{\alpha}, t_{+\infty}-t_{-\infty},\|\phi\|_{L i p}, \phi_{\infty}^{\prime}\right)
$$

it follows that $K_{\phi}$ depends continuously on $\phi$ in any norm in which these parameters are continuous. In particular, if $\phi$ is the perturbation of a fixed $\phi_{0}$, we can write

$$
K_{\phi}=\frac{1}{|\alpha|} K(\delta, \phi) \quad \text { where } \quad K(\delta, \phi)=O(1)
$$

Indeed, (20) yields $N_{\alpha}=O(1 /|\alpha|)$, so that (31) yields $K_{1}=O(1 /|\alpha|)$ and using

$$
\left(1+|\alpha|\|\phi\|_{L i p}\right)^{N_{\alpha}} \leq e^{|\alpha|\|\phi\|_{L i p} N_{\alpha}}=O(1),
$$

we see that (23) also gives $V_{\phi}=O(1 /|\alpha|)$. 


\section{INVERSION OF $\Delta_{\phi}$ IN OTHER NORMS.}

We now address the question: in which other norms is $\Delta_{\Phi}$ invertible? We consider only functions which are at least Lipshitz continuous, so that the results of the previous section apply. Our goal then is to identify those norms in which $\Delta_{\Phi}$ is invertible on its range,

$$
\|v\| \leq K\|w\| \quad \text { if } \quad \Delta_{\Phi} v=w .
$$

To be specific, let $\|\cdot\|$ denote the norm and let $X$ be the Banach space of Lipshitz functions on $\left[t_{-\infty}, t_{+\infty}\right]$ bounded in this norm; that is,

$$
X=\left\{v \in C^{0,1}\left[t_{-\infty}, t_{+\infty}\right],\|v\|<\infty\right\} .
$$

Our starting assumption is that the norm $\|\cdot\|$ respects composition, in the sense that

$$
\|v \circ \Psi\| \leq K_{0}\|v\|\|\Psi\| \quad \text { for some } K_{0},
$$

provided $\Psi:\left[t_{-\infty}, t_{+\infty}\right] \rightarrow\left[t_{-\infty}, t_{+\infty}\right]$ and $\Psi \in X$. In particular the shift operator $\mathcal{S}_{\Phi}$ is a bounded operator on $X$, whose norm is controlled by $\|\Phi\|$, and so $\Delta_{\Phi}: X \rightarrow X$ is a bounded operator,

$$
\left\|\Delta_{\Phi}\right\| \leq K_{0}\|\Phi\|+1 \text {. }
$$

The following theorem gives a sufficient condition for the inverse of $\Delta_{\Phi}$ to be bounded in a given norm.

Theorem 3. Assume the Banach space $X$ has a norm satisfying (36). The range of the operator $\Delta_{\Phi}: X \rightarrow X$ is the set $\mathcal{R}$ that consists of those functions $w \in X$ that satisfy (12), which implicitly implies $w\left(t_{ \pm \infty}\right)=0$. Moreover, if we can sum either of the series

$$
\sum_{k=0}^{\infty}\left\|\Phi^{k}\right\|<\infty, \quad \text { or } \quad \sum_{k=1}^{\infty}\left\|\Phi^{-k}\right\|<\infty,
$$

where as usual, $\Phi^{k}$ denotes $k$-fold composition, then the solution operator $\Delta_{\Phi}^{-1}$ is bounded on its domain. That is, there is a constant $K$ such that, if $w \in \mathcal{R}$ and $v$ satisfies

$$
\Delta_{\Phi} v=w, \text { then }\|v\| \leq K\|w\| .
$$

We regard (37) as a condition on $\Phi$ (or $\alpha \phi$ ) which implies that the equation can be solved in $X$ : that is, if the norm satisfies (36), then condition (37) is a condition only on the shift function which implies that the equation $\Delta_{\Phi}$ is invertible on its range in the space $X$.

Proof. Since the norm satisfies (36), the forward operator is bounded and maps $X \rightarrow X$. Since $X$ is a subset of the Lipshitz continuous functions, the range $\mathcal{R}$ consists of those elements of $X$ which satisfy our consistency conditions $w\left(t_{ \pm \infty}\right)=0$ and (12). 
We must show that if $w \in \mathcal{R}$, and if $v$ solves $\Delta_{\Phi} v=w$, then $\|v\| \leq K\|w\|$ for some $K$. Since the solution $v$ is given by a formula, namely (11), this bound becomes an explicit inequality. To prove the theorem, use the formula of (11) corresponding to the finite sum in (37), and set the corresponding $v\left(t_{+\infty}\right)$ or $v\left(t_{-\infty}\right)$ to zero. For definiteness, suppose that the first inequality in (37) holds, say

$$
\sum_{k=0}^{\infty}\left\|\Phi^{k}\right\|=M
$$

then set $v\left(t_{+\infty}\right)=0$ and use the first equation in (11) to define $v$. Using (36), it follows that

$$
\begin{aligned}
\|v\| & =\left\|\sum_{k=0}^{\infty} w \circ \Phi^{k}\right\| \\
& \leq \sum_{k=0}^{\infty} K_{0}\|w\|\left\|\Phi^{k}\right\| \\
& \leq K\|w\|,
\end{aligned}
$$

with $K=K_{0} M$, as required; the other case follows similarly.

In light of (37), the following corollary is immediate.

Corollary 5. If $K_{0}=1$ in (36), then a sufficient condition for the operator $\Delta_{\Phi}$ to be bounded is that for some $\eta>0$ and $k$ large enough, we have

$$
\left\|\Phi^{k+1}\right\| \leq(1-\eta)\left\|\Phi^{k}\right\|, \quad \text { or } \quad\left\|\Phi^{-k-1}\right\| \leq(1-\eta)\left\|\Phi^{-k}\right\| .
$$

Note that these bounds apply for the Lipshitz norm, cf. Theorem 1 and (24).

4.1. Bounds in the $C^{p}$ Norm. Because the kernel of $\Delta_{\Phi}$ is infinite dimensional in spaces of discontinuous functions, it is most natural to establish invertibility of in spaces in which the function and its derivatives are continuous. Here we prove invertibility of $\Delta_{\Phi}$ in $C^{p}$, the simplest such spaces.

Recall that for integers $p \geq 0$, we can write the $C^{p}$ norm as

$$
\|f\|_{p}=\sup _{t}|f(t)|_{p}
$$

where we have set

$$
|f(t)|_{p}:=\left|\left(f(t), f^{\prime}(t), \ldots, f^{(p)}(t)\right)^{T}\right|=\sup _{t} \sum_{j=0}^{p}\left|f^{(j)}(t)\right|,
$$

that is, $|f(t)|_{p}$ is a norm of the vector of derivatives up to $p$-th order. It is convenient to work with pointwise values of the iterates $\Phi^{k} t$. The point here is that the order $p$ is fixed, while the iteration number $k$ is large, so we wish to give an asymptotic description of $\frac{d^{p}}{d t^{p}} \Phi^{k} t$. We know that $\Phi^{k} t \rightarrow t_{+\infty}$ geometrically for large $k$, and we wish to leverage this to get bounds for $\left\|\Phi^{k}\right\|_{p}$ 
Lemma 5. For $p \geq 1$, the space $C^{p}\left[t_{-\infty}, t_{+\infty}\right]$, endowed with the norm $\|f\|_{p}=\sup _{t}|f(t)|_{p}$, is a Banach space containing the Lipshitz functions and for which the bound (36) holds.

Rather than treat the case of general $p$, we treat the case $p=3$, which illustrates the development for the case of general $p$, which in turn follows by induction. We show (37) holds for the norm $\|f\|_{p}$, as long as $\phi$ is itself $C^{p+1}$. The goal is to take advantage of the geometric convergence of $\Phi^{k} t$ to the limit $t_{ \pm \infty}$ as $k \rightarrow \pm \infty$.

To control the derivatives of $\Phi$, we introduce the following notation: for a given $t=t_{0}$, define

$$
t_{k}=\Phi^{k} t, \quad s_{k}=\left(\Phi^{k} t\right)^{\prime}, \quad r_{k}=\left(\Phi^{k} t\right)^{\prime \prime}, \quad \text { and } \quad q_{k}=\left(\Phi^{k} t\right)^{\prime \prime \prime} .
$$

We now describe the discrete dynamical system for the vector

$$
U_{k}=\left(t_{k}, s_{k}, r_{k}, q_{k}\right)^{T} .
$$

To begin, assume $z=z(t)$ is some given function, and compute

$$
\begin{aligned}
(\Phi z)^{\prime} & =\Phi^{\prime}(z) z^{\prime}=\left(1+\alpha \phi^{\prime}(z)\right) z^{\prime}, \\
(\Phi z)^{\prime \prime} & =\left(1+\alpha \phi^{\prime}(z)\right) z^{\prime \prime}+\alpha \phi^{\prime \prime}(z)\left(z^{\prime}\right)^{2}, \quad \text { and } \\
(\Phi z)^{\prime \prime \prime} & =\left(1+\alpha \phi^{\prime}(z)\right) z^{\prime \prime \prime}+3 \alpha \phi^{\prime \prime}(z) z^{\prime} z^{\prime \prime}+\alpha \phi^{\prime \prime \prime}(z)\left(z^{\prime}\right)^{3} .
\end{aligned}
$$

Now setting $z=\Phi^{k} t$ and using (38), we obtain the system

$$
\begin{aligned}
& t_{k+1}=t_{k}+\alpha \phi\left(t_{k}\right), \\
& s_{k+1}=\left(1+\alpha \phi^{\prime}\left(t_{k}\right)\right) s_{k}, \\
& r_{k+1}=\left(1+\alpha \phi^{\prime}\left(t_{k}\right)\right) r_{k}+\alpha \phi^{\prime \prime}\left(t_{k}\right) s_{k}^{2}, \\
& q_{k+1}=\left(1+\alpha \phi^{\prime}\left(t_{k}\right)\right) q_{k}+3 \alpha \phi^{\prime \prime}\left(t_{k}\right) s_{k} r_{k}+\alpha \phi^{\prime \prime \prime}\left(t_{k}\right) s_{k}^{3},
\end{aligned}
$$

which we regard as a discrete dynamical system,

$$
U_{k+1}=F\left(U_{k}\right)
$$

where $F$ is the RHS of (39). Note that this is a hierarchy, in that each successive equation beyond the nonlinear equation for $t_{k}$ is a linear inhomogeneous equation, once the previous components are given. It is clear that the system can be extended to arbitrary fixed values of $p$. Also, since each equation beyond the first is linear, solutions exist and remain bounded for all finite $k$.

It is easy to find the fixed points of (39) by directly finding the rest points of each equation in turn: the first equation yields $\phi(t)=0$, so $t=t_{ \pm \infty}$, and subsequent equations have trivial solutions because $\phi^{\prime}\left(t_{ \pm \infty}\right) \neq 0$, and the fixed points are

$$
U_{-\infty}:=\left(t_{-\infty}, 0,0,0\right)^{T} \text { and } U_{+\infty}:=\left(t_{+\infty}, 0,0,0\right)^{T} .
$$

We now show that, just as in the case for the scalar dynamical system for $t_{k}$, the rest points $U_{ \pm \infty}$ are a source and sink, respectively. 
Lemma 6. For $\phi \in C^{p+1}$ and $|\alpha|<1 / \sup \left|\phi^{\prime}(t)\right|$, there are constants $\epsilon_{\phi}$ and $\eta>0$, such that if

$$
\left|U-U_{+\infty}\right|<\epsilon_{\phi}, \quad \text { then }\left|F(U)-U_{+\infty}\right|<(1-\eta)\left|U-U_{+\infty}\right|,
$$

while also, if

$$
\left|U-U_{-\infty}\right|<\epsilon_{\phi}, \quad \text { then }\left|U-U_{-\infty}\right|<(1-\eta)\left|F(U)-U_{-\infty}\right| .
$$

Also, given any compact set $\mathcal{K}$, there is a constant $N_{\mathcal{K}}$ such that: if $U_{0} \in \mathcal{K}$ and $t_{0} \in\left[t_{-\infty}+\epsilon_{\phi}, t_{+\infty}-\epsilon_{\phi}\right.$, then

$$
\left|U_{k}-U_{+\infty}\right|<\epsilon_{\phi} \quad \text { and } \quad\left|U_{-k}-U_{-\infty}\right|<\epsilon_{\phi},
$$

for $k \geq N_{\mathcal{K}}$

This lemma states that the dynamical system (40) is contractive in a neighborhood of $U_{+\infty}$, and backwards contractive in a neighborhood of $U_{-\infty}$, and that given any bounded initial state, a finite number of forward or backward steps will lead into these neighborhoods.

Proof. We have already seen that $U_{-\infty}$ and $U_{+\infty}$ are fixed points of $F$, and so are rest points of the dynamical system. To linearize around these fixed points, we compute

$$
D F(U)=\left(\begin{array}{cccc}
1+\alpha \phi^{\prime} & 0 & 0 & 0 \\
\alpha \phi^{\prime \prime} s & 1+\alpha \phi^{\prime} & 0 & 0 \\
\alpha \phi^{\prime \prime} r+\alpha \phi^{\prime \prime \prime} s^{2} & 2 \alpha \phi^{\prime \prime} s & 1+\alpha \phi^{\prime} & 0 \\
\square & 3 \alpha \phi^{\prime \prime} r+3 \alpha \phi^{\prime \prime \prime} s^{2} & 3 \alpha \phi^{\prime \prime} s & 1+\alpha \phi^{\prime}
\end{array}\right),
$$

with $\square=\alpha \phi^{\prime \prime} q+3 \alpha \phi^{\prime \prime \prime} s r+\alpha \phi^{(4)} s^{3}$, where we note that this derivative is well defined because $\phi \in C^{p+1}$. Next, writing

$$
F(U)-F\left(U_{ \pm \infty}\right)=\int_{0}^{1} D F\left(U_{ \pm \infty}+\sigma\left(U-U_{ \pm \infty}\right)\right) d \sigma\left(U-U_{ \pm \infty}\right)
$$

the estimates follow provided the eigenvalues of $D F(U)$ are bounded by $1-\eta$ in a neighborhood of $U_{+\infty}$ and by $1 /(1-\eta)$ in a neighborhood of $U_{-\infty}$, respectively. Since $\alpha \phi^{\prime}\left(t_{+\infty}\right)<0$ and $\alpha \phi^{\prime}\left(t_{-\infty}\right)>0$, respectively, the eigenvalues at $U_{ \pm \infty}$ are of the right form, and by continuity we can choose $\epsilon_{\phi}>0$ and $\eta=O(\alpha)$ so that (41) and (42) hold.

We now verify (43) for the above chosen $\epsilon_{\phi}$ at $t_{+\infty}$, the case at $t_{-\infty}$ being similar. So let $\mathcal{K} \subset \mathcal{R}^{4}$ be compact. We show that there exists an $N_{\mathcal{K}} \in \mathcal{N}$ such that if $U_{0} \in \mathcal{K}$ and $t_{0} \geq t_{-\infty}+\epsilon_{\phi}$, then $\left|U_{k}-U_{\infty}\right|<\epsilon_{\phi}$ for $k \geq N_{\mathcal{K}}$. We define $N_{\mathcal{K}}$ by induction on the components in system (39), using the fact that system (39) is hierarchical. Let $(39) j$ denote the $j$ 'th equation in (39). We construct $N$ by induction on $j$. Since $\mathcal{K}$ is compact, there exists a constant $M_{\mathcal{K}}$ such that $U \in \mathcal{K}$ implies $|U|<M_{\mathcal{K}}$.

To start the induction, note that since $\alpha \phi^{\prime}<1$ and $\phi>0$ in $\left(t_{-\infty}, t_{+\infty}\right)$, (39) 1 implies that if $t_{k} \in\left(t_{-\infty}, t_{+\infty}\right)$, then $t_{k+1} \in\left(t_{-\infty}, t_{+\infty}\right)$, and $t_{k+1}>t_{k}$. Thus since $t_{0} \geq t_{-\infty}+\epsilon_{\phi},(39)_{1}$ together with the fact that the first five 
derivatives of $\phi$ are continuous, implies that there exists an $N_{1}$ such that if $k>N_{1}$, then

$$
\alpha\left|\phi^{\prime}\left(t_{k}\right)\right|>\frac{\alpha\left|\phi^{\prime}\left(t_{\infty}\right)\right|}{2}
$$

where $\phi^{\prime}\left(t_{\infty}\right)<0$, and

$$
\left|\phi^{(j)}\left(t_{k}\right)-\phi^{(j)}\left(t_{\infty}\right)\right|<\delta,
$$

for $\delta=\frac{\eta}{2}, j=1, \ldots, p=3$.

Consider now equation $(\sqrt[39]{39})_{2}$. Since $\left(1+\alpha \phi^{\prime}\right)<2$, it follows from $(\sqrt{39})_{2}$ that $s_{N_{1}} \leq 2^{n} s_{0}<2^{n} M_{\mathcal{K}}$. Thus by (44), (there is a mininum decrease in $s_{k}$ at each step), it follows that there exists $N_{2}$ such that $\left|s_{k}\right|<\epsilon_{\phi}$ for $k>N_{1}+N_{2}$.

Consider next equation (39) 3 . Since $\left(1+\alpha \phi^{\prime}\left(t_{k}\right)\right)<1-\frac{\eta}{2}$ for $k>N_{1}$ and $s_{k}^{2}$ decreases monotonically and quadratically to zero for $k>N_{1}+N_{2}$, it follows that $r_{N_{1}+N_{2}}<2^{N_{1}+N_{2}} M_{\mathcal{K}}$, and after $k>N_{1}+N_{2}, s_{k}$ monotonically decreases, it follows that there exists $N_{3}$ such that $\left|r_{k}\right|<\epsilon_{\phi}$ for $k>N_{1}+$ $N_{2}+N_{3}$.

For the fourth equation (39) 4 , note that the corrections to $q_{k+1}=(1+$ $\left.\alpha \phi^{\prime}\left(t_{k}\right)\right) q_{k}$ are quadratic in the variables prior to $q_{k}$, so by the same argument, there exists $N_{4}$ such that if $k>N \equiv N_{\mathcal{K}}=N_{1}+\cdots N_{4}$, then $\left|q_{k}\right|<\epsilon_{\phi}$, as claimed.

In summary, for the induction step, use that if $p$ is the variable on the LHS of the $p$ 'th equation, then by the hierarchical character of system(39), the $p$ 'th equation looks like $p_{k+1}=\left(1+\alpha \phi^{\prime}\left(t_{k}\right)\right) p_{k}$ plus corrections that at least quadratic in prior variables. Thus the corrections tend to zero so fast that a finite $N_{p}$ always exists to drive $p_{k}$ back to zero, overcoming the growth in $p_{k}$ during the first $N_{1}+\cdots N_{p-1}$ steps. This concludes the proof of Lemma 6.

Note that if $t_{0}=t_{-\infty}$ but $U_{0} \neq U_{+\infty}$, then the forward dynamical system has solutions which tend to infinity. Thus we do not have a uniform $N$ such that

$$
\left|U_{k}-U_{+\infty}\right|<\epsilon_{\phi}
$$

for $k>N$, without the condition that $t_{0}$ should be at least $\epsilon_{\phi}$ from $t_{ \pm \infty}$. The two cases in (39) suffice because the solvability condition implies we have both a backward and forward time formula for the solution $v$, c.f. (11).

We now use these properties of the dynamical system (39) to sum the norms of iterates as in (37).

Theorem 4. For integers $p \geq 1$ and fixed $\phi \in C^{p+1}$, there exists an $\alpha_{0}>0$ such that if $|\alpha| \leq \alpha_{0}$, then the operator $\Delta_{\Phi}: C^{p}\left[t_{-\infty}, t_{+\infty}\right] \rightarrow C^{p}\left[t_{-\infty}, t_{+\infty}\right]$ has a bounded inverse on its range, with bound

$$
\left\|\Delta_{\Phi}^{-1} w\right\|_{p} \leq \frac{K}{|\alpha|}\|w\|_{p},
$$


where the dependence of the constant $K$ is given by

$$
K \equiv K\left(\alpha, \delta, \epsilon_{\phi}, N_{\alpha}, t_{+\infty}-t_{-\infty},\|\phi\|_{p+1}\right) .
$$

Proof. It is convenient to use our previous estimate for the function, and estimate only the first through $p$-th derivatives here. For fixed $t_{0}$, we must bound one of the two sums

$$
\left|\sum_{j=0}^{\infty} w\left(\Phi^{j} t_{0}\right)\right|_{\hat{p}} \quad \text { or } \quad\left|\sum_{j=1}^{\infty} w\left(\Phi^{-j} t_{0}\right)\right|_{\hat{p}}
$$

where $|\cdot|_{\hat{p}}$ is the norm of the vector of the first through $p$-th derivatives,

$$
|f(t)|_{\hat{p}}:=\left|\left(f^{\prime}(t), \ldots, f^{(p)}(t)\right)^{T}\right|=\sup _{t} \sum_{j=1}^{p}\left|f^{(j)}(t)\right| .
$$

Also note that

$$
\left|\sum w\left(\Phi^{j} t\right)\right|_{\hat{p}} \leq K_{0}\|w\|_{p}\left|\sum \Phi^{j} t\right|_{\hat{p}}=K_{0}\|w\|_{p}\left|\sum \hat{U}_{k}\right|,
$$

where $\hat{U}_{k}=\left(s_{k}, r_{k}, q_{k}\right)^{T}$, see (38). We thus need a uniform bound on either of the sums

$$
\left|\sum_{j=0}^{\infty} \hat{U}_{j}\right| \text { or }\left|\sum_{j=1}^{\infty} \hat{U}_{-j}\right| .
$$

Since $\phi \in C^{p+1}$, each derivative is uniformly bounded and so there is a compact set $\mathcal{K}$ such that $U_{0} \in \mathcal{K}$ uniformly in $t_{0}$. Using Lemma 6, we know that for $k \geq N_{\mathcal{K}}$, either $U_{k}$ is close to $U_{+\infty}$ or $U_{-k}$ is close to $U_{-\infty}$, for any $k \geq N_{\mathcal{K}}$. In the first case, we have $\left|\hat{U}_{k+1}\right| \leq(1-\eta)\left|\hat{U}_{k}\right|$ for all $k \geq N_{\mathcal{K}}$, so that

$$
\begin{aligned}
\left|\sum_{j=0}^{\infty} \hat{U}_{j}\right| & \leq\left|\sum_{j=0}^{N_{\mathcal{K}}-1} \hat{U}_{j}\right|+\sum_{j=N_{\mathcal{K}}}^{\infty}(1-\eta)^{j-N_{\mathcal{K}}}\left|\hat{U}_{N_{\mathcal{K}}}\right| \\
& \leq\left|\sum_{j=0}^{N_{\mathcal{K}}-1} \hat{U}_{j}\right|+\frac{\left|\hat{U}_{N_{\mathcal{K}}}\right|}{\eta} \leq \frac{K_{1}}{\eta}
\end{aligned}
$$

and similarly for the second case, $\left|\hat{U}_{-k-1}\right| \leq(1-\eta)\left|\hat{U}_{-k}\right|$ for $k \geq N_{\mathcal{K}}$, so that

$$
\begin{aligned}
\left|\sum_{j=1}^{\infty} \hat{U}_{-j}\right| & \leq\left|\sum_{j=1}^{N_{\mathcal{K}}-1} \hat{U}_{-j}\right|+\sum_{j=N_{\mathcal{K}}}^{\infty}(1-\eta)^{j-N_{\mathcal{K}}}\left|\hat{U}_{-N_{\mathcal{K}}}\right| \\
& \leq\left|\sum_{j=1}^{N_{\mathcal{K}}-1} \hat{U}_{-j}\right|+\frac{\left|\hat{U}_{-N_{\mathcal{K}}}\right|}{\eta} \leq \frac{K_{1}}{\eta} .
\end{aligned}
$$

Now taking the supremum over all $t=t_{0}$ in (46) completes the proof. 


\section{REFERENCES}

[1] Marek Kuczma, Marek, Bogdan Choczewski, Roman Ger, Iterative Functional Equations, Cambridge University Press, Jul 27, 1990 - Mathematics - 552 pages

[2] A. Majda, Compressible Fluid Flow and Systems of Conservation Laws in Several Space Variables, Springer-Verlag, Applied Mathematical Sciences, 53, 1984.

[3] J. Moser, A new technique for the construction of solutions of nonlinear differential equations, Proc. Nat. Acad. Sci., 47, 1961, pp 1824-1831.

[4] B. Temple and R. Young, A paradigm for time-periodic sound wave propagation in the Compressible Euler Equations, Meth Appl of Anal, Vol. 16, 3 2009, pp 341-364.

[5] B. Temple and R. Young, A Liapunov-Schmidt Reduction for Time-Periodic Solutions of the Compressible Euler Equations, Meth Appl Anal Vol. 17, 3, 2010, pp 225-262.

[6] B. Temple and R. Young, Time-periodic linearized solutions of the compressible Euler equations and a problem of small divisors, SIAM Journal of Math Anal, Vol 43, 12011 , pp 1-49.

[7] B. Temple and R. Young, A Nash-Moser framework for finding periodic solutions of the compressible Euler equations, J Sci Comp, Vol. 59, No. 1 (2014).

[8] R. Young, Differentiability of Nonlinear Hyperbolic Evolution Operators, (Preprint).

Department of Mathematics, University of California, Davis, CA 95616

Department of Mathematics and Statistics, University of Massachusetts, AmHerst, MA 01003 